\title{
An analysis of the pharmacological management of respiratory tract infections in pediatric in-patients at a tertiary care teaching hospital
}

\section{Geetha S. lyer, Prakruti P. Patel, Jigar R. Panchal', R. K. Dikshit}

Departments of Pharmacology, B J Medical College, Ahmedabad, ${ }^{1}$ Pharmacology, Government Medical College, Vadodara, Gujarat, India

Address for the Correspondence:

Dr. Geetha S. lyer, Department of Pharmacology,

B J Medical College, Ahmedabad - 380 016, Gujarat, India E-mail: drgeetha1985@gmail.com

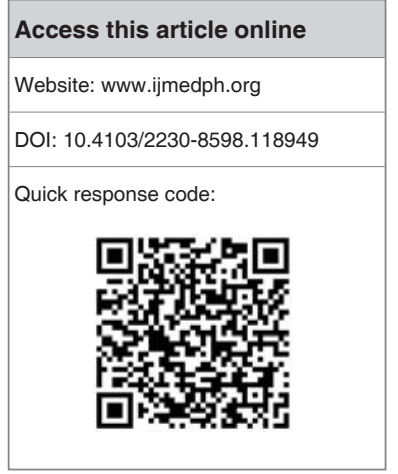

Objectives: To analyze the pharmacological management of respiratory tract infections in hospitalized pediatric patients. The economic burden of the disease and the adverse drug reactions occurring during the course of treatment have also been studied. Materials and Methods: It was a prospective, longitudinal, and observational study, carried out over a period of one-and-a-half years. The patients, aged one month to twelve years, diagnosed with respiratory tract infections (RTI), as confirmed by a pediatrician, were enrolled. Modified Kunin's criteria along with the guidelines set by the Indian Academy of Pediatrics (IAP) were followed for assessing the appropriateness of the antibacterials prescribed. The adverse drug reactions occurring during the course of the treatment were noted and the economic burden of the disease was calculated as direct and indirect costs. Results: A total of 201 patients were enrolled, of whom $46 \%$ were in the age group of one month to one year. The most common diagnosis was pneumonia $(76 \%)$. The number of drugs and antibacterials prescribed per patient were $4.88 \pm 1.57$ and $1.85 \pm 0.86$, respectively. Antibacterials $(37.7 \%)$, intravenous fluids $(17.2 \%)$, respiratory medicines $(16.6 \%)$, and analgesic/antipyretics $(16.5 \%)$ were the common drug groups prescribed. Among the antibacterials, amoxicillin with clavulanic acid $(47 \%)$ and cefotaxime (22\%) were frequently prescribed. Appropriate antibacterial therapy was given in $42 \%$ of the cases. Salbutamol and a combination of Levosalbutamol and Ipratropium Bromide were the commonly prescribed respiratory medicines. Three adverse drug reactions (ADRs) were observed in indoor patients, all due to antibacterials. The economic burden of the disease was calculated to be Rs. 4925.14 or US\$109.9 per patient. Conclusion: An overuse of antibacterials and respiratory medicines was seen in the study. Emphasis on proper diagnosis and treatment, education and availability of locally effective guidelines may help in a better and judicious use of drugs in children.

Key words: Adverse drug reactions, antibacterials, drug utilization, modified Kunin's criteria, respiratory medicines

\section{INTRODUCTION}

Respiratory infections, especially pneumonia, are an important cause of morbidity and mortality in children. ${ }^{[1]}$ In India, pneumonia is responsible for $20 \%$ of the under-five mortality, as compared to only $3 \%$ in the United States (US) and United Kingdom (UK). ${ }^{[2]}$ India also has the unfortunate distinction of having the highest predicted number of new pneumonia episodes (43 million per year), as estimated by the World Health Organization (WHO), in the year 2000. ${ }^{[1]}$ One of the 'Millennium Development Goals' is to reduce the under-five and infant mortality rate to one-third by 2015, as compared to 1990. With less than five years left to fulfill this goal, the cavalry is sadly lagging behind in implementation, with an additional 13.2 million deaths expected between 2010 and 2015..$^{[3]}$

The alarming rise in mortality can be offset by proper diagnosis and treatment along with the establishment of preventive measures like universal immunization. Although the practice guidelines are available for all respiratory infections, the extent of their implementation and effectiveness has not yet been analyzed. ${ }^{[4]}$ Also most of the guidelines are based on the etiology (viral, bacterial), but in most developing countries, including India, the therapy is usually empirical. ${ }^{[5]}$ The paucity of guidelines 
for the Indian scenario, along with the documented irrational use of antibacterials in respiratory infections, ${ }^{[6]}$ has led to consequences like widespread drug resistance. However, without the knowledge of how drugs are being prescribed, it is difficult to initiate a discussion on rational drug use and to suggest measures to change the prescribing habits for the better. Drug utilization research is an important tool, defined by the WHO, in 1977, as, "the marketing, distribution, prescription, and use of drugs in a society, with special emphasis on the resulting medical, social, and economic consequences". Given the high incidence of respiratory tract infections in the pediatric age group and lack of studies in this field, particularly in hospitalized patients, the present study has been undertaken. The aim of the study is to analyze the utilization pattern of the drugs prescribed in pediatric patients admitted with respiratory tract infections to a tertiary care, teaching hospital, as also the economic burden and adverse drug reactions occurring during the course of treatment. We have also made an attempt to assess the appropriateness of the antibacterials prescribed.

\section{MATERIALS AND METHODS}

This was a prospective, observational, single-center study, carried out over a period of 18 months (November 2008 to April 2010) among the patients hospitalized in the pediatric ward under a single unit of a tertiary care teaching hospital. Permission from the Medical Superintendent and the Head of the respective unit was obtained before conducting the study. A pilot study of one month was undertaken to test the predesigned case record form (CRF), which was then modified accordingly. Patients, aged one month to twelve years, whose primary diagnosis was respiratory tract infection (RTI), as confirmed by a pediatrician, were enrolled, and a written consent was obtained from their parents/guardians. The demographic details and information regarding diagnosis and treatment were recorded in the CRF. Modified Kunin's criteria ${ }^{[7]}$ were used to assess the appropriateness of the prescribed antibacterials.

The cases are categorized as follows:

I. The indication and protocol of the antibacterial therapy are appropriate

II. Agree with the use of antibacterial therapy, the protocol is probably appropriate. Microbiological evidence is lacking to confirm the diagnosis

III. Agree with the use of antibacterial therapy, but a different drug (less expensive, less toxic, narrower spectrum, other combination) will be appropriate

IV. Agree with the use of antibacterial therapy but a modified dose, interval, duration or route of administration will be appropriate

V. Disagree with the use of antibacterial therapy, administration is unjustified.

Categories I and II indicated 'appropriate therapy', categories III and IV indicated that there was some 'major deficiency' in the choice or use of antibacterials, and category $\mathrm{V}$ indicated 'unnecessary' antibacterial use. The choice of antibacterials was compared with the guidelines put forth by the Indian Academy of Pediatrics. ${ }^{[8]}$ Drug Utilization (DU) $90 \%$ Index was applied for the prescribed antibacterials. The treating pediatricians and the patients were requested to report any suspected adverse drug reactions (ADRs) THAT were recorded in an ADR reporting form. Causality assessment was done using THE WHO-Uppsala Monitoring Center (UMC) scale ${ }^{[9]}$ and Naranjo's algorithm. ${ }^{[10]}$ The severity and preventability of the ADRs were also assessed by using the Hartwig and Siegle scale ${ }^{[11]}$ and the modified Schumock and Thornton criteria, ${ }^{[12]}$ respectively. The economic burden of the disease was calculated under direct and indirect costs. Direct cost referred to the cost of the drugs prescribed, the cost incurred by the hospital for patient admission, and the cost of transportation, food, and lodging of the attendants (parents/guardians). Indirect cost comprises of the loss of wages as incurred by the parent or guardian. ${ }^{[13]}$ Cost of medication was obtained from the hospital (if supplied by the hospital) or private pharmacies (if unavailable in the hospital pharmacy). The data collected was analyzed with the help of the Microsoft Excel spreadsheet 2007.

\section{RESULTS}

A total of 201 indoor patients were studied. The demographic details and diagnosis are shown in Table 1 . The mean age was $2.26 \pm 2.42$ years (range 1.5 months to 11 years). About half the patients $(45.8 \%)$ were less than one-year-old and the number of boys $(69.6 \%)$ was almost twice the number of girls. Almost two-thirds of the patients were adequately immunized as per their age. The most common presenting complaints were cough $(97 \%)$, fever $(92 \%)$, and breathlessness (50\%). Routine investigations like complete blood counts and chest X-ray were conducted in all the patients, while specialized investigations like ultrasonography (6), computed

\begin{tabular}{lc}
$\begin{array}{l}\text { Table 1: Demographic characteristics of pediatric } \\
\text { inpatients with respiratory tract infection }(\boldsymbol{n}=201)\end{array}$ \\
$\begin{array}{l}\text { Patient characteristics } \\
\text { No. of patients (\%) }\end{array}$ \\
\hline Age & $92(46)$ \\
$>1$ month to $\leq 1$ year & $72(36)$ \\
$>1$ year to $\leq 3$ years & $13(7)$ \\
$>3$ years to $\leq 5$ years & $24(12)$ \\
$>5$ years & \\
Gender & $140(70)$ \\
Boys & $61(30)$ \\
Girls & \\
Immunization status & $133(66)$ \\
Complete & $60(30)$ \\
Partial & $7(4)$ \\
Unimmunized & $1(0.5)$ \\
Unknown & \\
Diagnosis (according to ICD 10 classification) & $154(77)$ \\
Lobar pneumonia, organism unspecified (J18.1) & $14(7)$ \\
Acute bronchiolitis, unspecified (J21.9) & $12(6)$ \\
Pneumonia with complications (J85.1, J 91) & $21(10)$ \\
Others* & \\
\hline
\end{tabular}

* Others include laryngotracheobronchitis, tonsillopharyngitis, non-specific upper respiratory tract infection (URTI), and progressive pulmonary disease, ICD= International classification of diseases 
tomography (CT) scan of the thorax (4), and Montoux test (3), were conducted, if needed. A majority of the patients were diagnosed with pneumonia $(76.6 \%)$ followed by bronchiolitis (6.9\%). Anemia (61\%) was the most common concurrent condition followed by gastrointestinal complaints (5\%) and congenital heart disease (4\%). The average duration of admission was $6.49 \pm 3.23$ days and patients with complicated pneumonia were hospitalized for a longer duration (up to 21 days).

A total of 982 prescriptions were given to 201 indoor patients. The average number of drugs prescribed per encounter was $4.88 \pm 1.57$ (range 2-12). The 982 drugs were prescribed as 1028 formulations in 46 cases; intravenous chloroquine and paracetamol were replaced by their oral formulation, as the patients' condition improved. Out of the 1028 formulations, 477 (46.4\%) and $551(53.6 \%)$ were prescribed by brand and generic names, respectively. About $70 \%$ of the drugs were supplied by the hospital, while the rest were bought from private pharmacies. The most commonly prescribed drug groups were antibacterials (37.7\%) followed by intravenous fluids $(17.2 \%)$, respiratory medicines (16.6\%), and analgesic/antipyretic drugs (16.5\%) [Table 2]. Nutritional supplements $(6.7 \%)$, steroids $(1.2 \%)$, and miscellany, including antimalarials, anthelmintics, antiemetics, and $\mathrm{H}_{2}$ blockers $(4 \%)$, were also prescribed. Respiratory medicines were the drugs most frequently (86\%) prescribed by brand names followed by nutritional supplements $(74.2 \%)$, while intravenous fluids $(100 \%)$ were most frequently prescribed by generic names followed by analgesic/antipyretic drugs (70\%). The details of antibacterials and respiratory medicines are given in Table 2 .

\section{Antibacterials}

Antibacterials were prescribed in 370 (38\%) prescriptions, of which amoxicillin/clavulanic acid (47\%) and cefotaxime (22\%) were the most common. Almost half $(50.3 \%)$ of the antibacterials were prescribed by their brand name. Five antibacterials, namely amoxicillin/clavulanic acid, cefotaxime, amikacin, ceftriaxone, and ampicillin made up the DU 90\% index. The average number of antibacterials prescribed per patient was $1.85 \pm 0.86$ (range 1-5) with the majority $(125 / 201)$ receiving two or more antibacterial agents during the course of their hospitalization $(97,13,14$, and 1 patient received $2,3,4$, and 5 antibacterials, respectively).

\begin{tabular}{|c|c|c|c|c|}
\hline \multirow[t]{2}{*}{ Drugs (ATC classification) } & \multicolumn{3}{|c|}{ Age groups } & \multirow[t]{2}{*}{ Total } \\
\hline & 1 month to 1 year & 1 year to 5 years & $>5$ years & \\
\hline \multicolumn{5}{|l|}{ Antibacterials (J01) } \\
\hline Crystalline penicillin & 0 & 0 & 1 & 1 \\
\hline Ampicillin & 15 & 3 & 0 & 18 \\
\hline Amoxicillin+Clavulanic acid & 76 & 76 & 23 & 175 \\
\hline Cefotaxime & 44 & 33 & 6 & 83 \\
\hline Ceftriaxone & 13 & 15 & 1 & 29 \\
\hline Ceftazidime & 2 & 2 & 0 & 4 \\
\hline Erythromycin & 0 & 1 & 2 & 3 \\
\hline Azithromycin & 1 & 1 & 2 & 4 \\
\hline Amikacin & 10 & 11 & 8 & 29 \\
\hline Vancomycin & 7 & 11 & 0 & 18 \\
\hline Metronidazole & 0 & 3 & 0 & 3 \\
\hline Levofloxacin & 0 & 0 & 2 & 2 \\
\hline Ofloxacin & 0 & 1 & 0 & 1 \\
\hline \multicolumn{5}{|l|}{ Respiratory system } \\
\hline Nasal preparations (R01) & 4 & 0 & 0 & 4 \\
\hline Adrenaline & 2 & 2 & 0 & 4 \\
\hline Salbutamol & 38 & 35 & 5 & 78 \\
\hline Ipratropium & 3 & 0 & 0 & 3 \\
\hline Theophylline & 2 & 1 & 0 & 3 \\
\hline Ipratropium+Levosalbutamol & 23 & 19 & 2 & 44 \\
\hline Theophylline+Salbutamol & 1 & 0 & 0 & 1 \\
\hline Pheniramine & 0 & 1 & 0 & 1 \\
\hline \multicolumn{5}{|l|}{ Cough and cold preparations (R05) } \\
\hline Bromhexine & 0 & 0 & 1 & 1 \\
\hline Dextromethorphan & 2 & 3 & 0 & 5 \\
\hline Antihistaminic+Antitussive+Decongestant ${ }^{*}$ & 8 & 4 & 2 & 14 \\
\hline Antihistaminic+Antitussive+Decongestant+Menthol ${ }^{*}$ & 0 & 2 & 1 & 3 \\
\hline Mucolytic+Expectorant+Bronchodilator+Menthol* & 0 & 1 & 0 & 1 \\
\hline Mucolytic+Bronchodilator* & 0 & 1 & 0 & 1 \\
\hline
\end{tabular}

*Indicates irrational fixed dose combination, ATC= International classification of diseases 
To assess the appropriateness of antibacterial use, guidelines set by the Indian Academy of Pediatrics and the modified Kunin's criteria $^{[7]}$ were used. It was observed that all patients (201) received antibacterials, irrespective of the laboratory results and chest $\mathrm{X}$-ray. The appropriateness of antibacterial use is described in Table 3. Appropriate therapy (criteria I and II) was given in $42.3 \%$ of the patients. In almost half the patients (48.7\%), a major deficiency in choice or use of antibacterials was noted (inappropriate treatment, criteria III and IV), and 8.9\% received unnecessary antibacterial treatment (criteria V). In 93 cases, a penicillin (ampicillin or amoxicillin + clavulanic acid) and a third generation cephalosporin (cefotaxime or ceftriaxone) were prescribed concomitantly. Amoxicillin + clavulanic acid and amikacin were prescribed together in 14 cases, while in seven cases, vancomycin and a third generation cephalosporin were used simultaneously.

There were 21 instances where the antibacterials were changed. Out of these, in 17 cases, a change was made after 48 hours, as no improvement took place clinically or on ultrasonography/CT scan. In three cases, the reason for change was not clear and in one case, the hospital supply of the drug was exhausted.

\section{Respiratory medicines}

A total of 163 prescriptions were made for respiratory medicines (RM), which mainly included cough and cold preparations (30) and bronchodilators (133) [Table 2]. The commonly used drugs were salbutamol and a combination of levosalbutamol and ipratropium bromide. Prescribing by brand name was most frequently observed in RMs (88\%). Fixed dose combinations (FDCs) constituted $43.4 \%$ of all prescriptions of RMs, while the rest were single drugs. A combination of antihistaminic, antitussive, and a nasal decongestant was the most common cough and cold preparation prescribed, followed by an antitussive alone. From Table 2, it is seen that almost $50 \%$ of the prescriptions for RMs were given to children below the age of one year. It was observed that bronchodilators were prescribed in all patients with a wheeze and those diagnosed with bronchiolitis. A combination of ipratropium bromide and levosalbutamol was noted to be prescribed predominantly in patients with symptoms that were severe (antimalarials, anthelmintics, antiemetics, and $\mathrm{H}_{2}$ blockers (4\%), intercostal and sternocostal indrawing, respiratory rate $>60$ ).

\section{Other medications}

It was observed that paracetamol (162/162) was the only analgesic/antipyretic prescribed. Isolyte-P (169/169) was the only intravenous fluid prescribed. Of all the prescriptions for nutritional supplements, $71 \%$ were fixed-dose combinations, the number of constituents ranging from 3-20. Folic acid, ferrous salts, and zinc were the commonly prescribed single drugs. Out of 12 prescriptions of steroids, six were in acute laryngotracheobronchitis and bronchiolitis each. Chloroquine, albendazole, ranitidine, domperidone, and ondansetron were the other prescribed drugs. Drugs like analgesic/antipyretics, $\mathrm{H}_{2}$ blockers, and antimalarials were given symptomatically by the right route and in appropriate doses.

\section{World health organization core indicators}

As per WHO core indicators, it was observed that polypharmacy was widely practiced. Antibacterials were prescribed in all patients. Similarly, almost all patients were prescribed injections. Only half the drugs were prescribed by their generic names. A majority of the drugs prescribed were from the National Essential Medicines List (EML) 2011 (75\%) and the WHO EML 2010 (70\%). An analysis of drugs prescribed is given in Table 4.

\section{Adverse drug reactions}

Only three ADRs were reported during the study, all of which were due to antibacterials (two cases of diarrhea due to ampicillin and one case of rash due to vancomycin). The causality for all three ADRs was 'possible' by the WHO-UMC scale and 'probable' by Naranjo's algorithm. They were mild in severity and preventable in nature.

\section{Cost of treatment}

The direct cost included the cost incurred by the hospital, cost of the prescribed drugs, intravenous fluids, medical supplies, and transportation to hospital. ${ }^{[13]}$ The average hospital expenditure incurred per patient per day of admission was Rs. 527.29. Since average hospitalization was for 6.49 days, the total hospital expenditure per patient was Rs. 3422.11. The cost of the drugs

\begin{tabular}{|c|c|c|}
\hline Kunin's criteria & No. of patients (\%) & Justification \\
\hline $\begin{array}{l}\text { Criteria I - Agree with the use of antimicrobial therapy, the } \\
\text { protocol is appropriate }\end{array}$ & $0(0)$ & $\begin{array}{l}\text { Laboratory reports - unable to conclude whether the } \\
\text { infection was bacterial or viral, treatment empirical }\end{array}$ \\
\hline $\begin{array}{l}\text { Criteria II - Agree with the use of antimicrobial therapy, the } \\
\text { protocol is probably appropriate, but a microbiology report } \\
\text { is missing, to classify the protocol in another category }\end{array}$ & $85(42)$ & $\begin{array}{l}\text { Microbiology testing was not performed to confirm } \\
\text { diagnoses. Clinical improvement speaks in favor of } \\
\text { correct empiric treatment }\end{array}$ \\
\hline $\begin{array}{l}\text { Criteria III - Agree with the use of antimicrobial therapy, } \\
\text { but a different antimicrobial is preferred }\end{array}$ & $97(48)$ & $\begin{array}{l}\text { Too many antibacterials were given concomitantly, } \\
\text { the combination of antibacterials was not synergistic } \\
\text { or antibiotic cover was inadequate in some cases }\end{array}$ \\
\hline $\begin{array}{l}\text { Criteria IV - Agree with the use of antimicrobial therapy, } \\
\text { but a modified dose, interval, duration or route of } \\
\text { administration is preferred }\end{array}$ & $1(0.5)$ & $\begin{array}{l}\text { A patient was given intravenous antibacterial when } \\
\text { patient was able to take orally }\end{array}$ \\
\hline $\begin{array}{l}\text { Criteria V - Disagree with the use of antimicrobial } \\
\text { therapy, administration is unjustified }\end{array}$ & $18(9)$ & Infections viral in origin, antibacterials not required \\
\hline
\end{tabular}




\begin{tabular}{|c|c|}
\hline WHO core indicators & $\begin{array}{l}\text { No. of patients } \\
\text { (\%) }\end{array}$ \\
\hline $\begin{array}{l}\text { Number of drugs prescribed per encounter } \\
\text { (mean } \pm \text { SD) }\end{array}$ & $4.88 \pm 1.57$ \\
\hline Number of drugs prescribed by generic name (\%) & $551(53.6)$ \\
\hline $\begin{array}{l}\text { Number of encounters resulting in the prescription } \\
\text { of an antibacterial (\%) }\end{array}$ & $201(100)$ \\
\hline $\begin{array}{l}\text { Number of encounters resulting in the prescription } \\
\text { of an injection (\%) }\end{array}$ & $200(99.5)$ \\
\hline $\begin{array}{l}\text { Number of drugs prescribed from the National } \\
\text { Essential Medicines List } 2011(\%)\end{array}$ & $735(75)$ \\
\hline $\begin{array}{l}\text { Number of drugs prescribed from WHO EML } \\
2010(\%)\end{array}$ & $689(70.2)$ \\
\hline
\end{tabular}

prescribed per patient was Rs. 341.88, for the medical supplies it was Rs. 20.55, and the cost of transportation per patient was Rs. 187.6. Hence, the direct cost per indoor patient amounted to a total of Rs. 3972.14. The indirect cost per indoor patient, that is, loss of wages incurred by the parents/guardians ${ }^{[13]}$ was Rs. 953. The total economic impact per patient amounted to Rs. 4925.14 or US\$109.9. The total impact of respiratory infections in all 201 patients was Rs. $989,953.14$ or US\$22,097.2.

\section{DISCUSSION}

The present study was done with an intention of analyzing the pharmacological management of respiratory tract infections in hospitalized children. The majority of enrolled patients were less than one year of age, which may be due to increased susceptibility to infections during the weaning period. The most frequent presenting complaints were cough, fever, and breathlessness, signifying the serious condition of the patient, requiring hospitalization. The common diagnoses were pneumonia and bronchiolitis, which have been noticed in Brazil, where nearly $30 \%$ of the admissions were due to pneumonia. ${ }^{[14]}$ No distinction was made between bacterial and viral etiologies in the diagnoses. This is important as viral pneumonia as well as bronchiolitis usually do not require antibacterial treatment.

A great deal of polypharmacy was evident as the average number of drugs prescribed per patient was nearly five (range 2-12). Antibacterials were observed to be the most frequently prescribed drugs, as was also observed in a study conducted in western Nepal, ${ }^{[15]}$ followed by analgesic/antipyretics, intravenous fluids, and RMs. Amoxicillin with clavulanic acid, cefotaxime, ceftriaxone, ampicillin, and amikacin constituted $90 \%$ of all the antibacterials prescribed. This finding is comparable to other studies reported from Kathmandu ${ }^{[16]}$ and Palestine, ${ }^{[17]}$ where either penicillin or cephalosporin were the most frequently prescribed antibacterials for pediatric inpatients. In these studies, older penicillin was used more frequently (crystalline penicillin and ampicillin), which can be attributed in part to the variation in the local resistance pattern. Also these studies were conducted four to six years earlier. Prescription of about two antibacterials on an average was a norm. Clearly the hassle of identifying the organism was avoided and several antibacterials were prescribed concomitantly to cover all possible organisms leading to greater chances of adverse drug reactions, drug interactions, development of resistance, increased cost of therapy, and so on. ${ }^{[18]}$

The appropriateness of the antibacterials prescribed was assessed by the modified Kunin's criteria. ${ }^{[7]}$ Appropriate therapy was given in $42 \%$ of the patients only. No case could be classified under criteria I, as no confirmatory laboratory reports were conducted to confirm the etiology. The cases falling under criteria II (42\%) received empirical therapy, with an acceptable antibacterial regime, but microbiological evidence confirming the diagnosis was lacking. In almost half the patients, either the prescribed antibacterial combinations were not synergistic, more than the required number of drugs were prescribed, or the spectrum of antibacterial cover was inadequate (in infants less than two months of age, where pneumonia is to be treated as septicaemia). Among the combinations, amoxicillin/clavulanic acid and cephalosporin have been frequently prescribed concomitantly, which is not justified, as their spectrum overlaps considerably with the difference being that cefotaxime is active against a few anaerobes also. Other combinations used were amoxicillin/clavulanic acid with amikacin, and cephalosporin with vancomycin. In 18 patients, the use of antibacterials was not justified, as the conditions were predominantly viral and did not require antibacterial therapy (criteria V). It was observed that antibacterials were prescribed in bronchiolitis where they have a limited value. ${ }^{[19]}$ Similar observations have been made before. ${ }^{[17]}$

Salbutamol and a combination of levosalbutamol and ipratropium bromide were the most common respiratory medicines prescribed. Use of bronchodilators in pneumonia and bronchiolitis is not well established. ${ }^{[20]}$ These drugs were used to provide symptomatic relief, particularly in those cases where a significant amount of wheeze is seen. It was also observed that almost $50 \%$ of the respiratory medicines were given to children below one year of age, which included cough and cold combinations. A Cochrane review of the efficacy of these combinations concluded that they were no more effective than placebo in children. ${ }^{[21]}$

The US FDA has banned the use of these medicines in children less than two years of age. ${ }^{[22]}$ The threat of overdose, combined with their lack of proven benefit in children does not support their use for relieving respiratory symptoms. Symptomatic and supportive therapy in the form of antipyretics and intravenous fluids has been used appropriately in these patients. Steroids have been prescribed only in cases of acute laryngotracheobronchitis (reduces laryngeal edema) and lobar pneumonia (ineffective). ${ }^{[23]}$

It should be mentioned that the ADRs seen in our study were caused by antibacterials, as was also seen in Spain, ${ }^{[24]}$ Brazil,${ }^{[25]}$ and Italy, ${ }^{[26]}$ thus substantiating the widely acknowledged fact that irrational use of antibacterials led to adverse drug reactions. A disturbing fact noted was that all ADRs were preventable in nature (two definitely 
and one probably), as either the causal drugs were not indicated in the patients or preventive measures could have been taken. Lack of suspicion that a complaint could be due to an ADR might be the reason for the low incidence of ADRs noted in our study. Even the patients themselves might not understand the correlation of a particular complaint (ADR) and the drug, and hence, not bring the reaction to the notice of the pediatrician.

The burden of the disease in indoor patients was Rs. 4925.14 or US \$ 109.9 per patient. Although significant, it was still less compared to other countries. In Germany, the total cost per hospitalized pediatric patient with community-acquired pneumonia was found to be $€ 2579$ (Rs. 153,778), ${ }^{[27]}$ while in Pakistan the average cost per episode ranged from $\$ 22$ to $\$ 142$ depending on the severity of the disease. ${ }^{[28]} \mathrm{A}$ difference in the healthcare policies between countries (reimbursements, higher monetary value for productivity) and nature of the study (our study is prospective, while most of other studies were retrospective) may account for this discrepancy.

Considering the high disease burden, the UNICEF and WHO have jointly developed a Global Action Plan for the prevention and control of Pneumonia (GAPP) ${ }^{[3]}$, in 2009, which has focused on developing countries and allocates monetary funds for its control. Drug Utilization Studies can help us facilitate the rational use of medicines and ensure the prudent use of the available resources. ${ }^{[2]}$ As seen in our study, antibacterials and respiratory medicines are often used indiscriminately. Education of the prescribers as well as the caregivers is imperative. Even as the heavy workload in Indian hospitals may be cited as a reason for the inability of doctors to communicate effectively with the parents, an effort must always be made in this regard, particularly with the help of paramedical workers.

This study has found the need for a standard treatment guideline for our own hospital, taking into account the local sensitivity pattern of the organisms. Although culture and antibiotic susceptibility were not performed in our study to confirm the rationality of the antibacterial used, the present study serves to highlight the current treatment practice of these infections in our hospital and pave the way for further interventions that can help implement the rational use of medicines.

\section{REFERENCES}

1. Rudan I, Boschi-Pinto C, Biloglav Z, Mulholland K, Campbell H. Epidemiology and etiology of childhood pneumonia. Bull World Health Organ 2008;86:408-16.

2. World Health Organization. World Health Statistics 2010. France: WHO; 2010.

3. United Nation's Children Emergency Fund, World Health Organization. Technical Consensus Report. Global action plan for the prevention and control of pneumonia. Report of an informal consultation. UNICEF, 2009.

4. Worrall G, Chaulk P. Hope or experience? Clinical practice guidelines in family practice. J Fam Pract 1996;42:353-6.

5. Hart CA, Kariuki S. Antimicrobial resistance in developing countries. BMJ 1998;317:647-50

6. Ashraf $\mathrm{H}$, Handa S, Khan NA. Prescribing pattern of drugs in outpatient department of child care centre in Moradabad city. Int J Pharm Sci Rev Res 2010;3:1-5.

7. Vlahovic-Palcevski V, Francetic I, Palcevski G, Novak S, Abram M, Bergman U. Antimicrobial use at a university hospital: Appropriate or misused? A qualitative study. Int J Clin Pharmacol Ther 2007;45:169-74.
8. Ganguly N. Lower respiratory tract infections. In: Ghosh TK, Yewale V, Parthasarthy A, Shah NK, editors. IAP speciality series on Pediatric Infectious Diseases (Under IAP Action Plan 2006). New Delhi: Jaypee Publishers; 2006. p. 221-35.

9. World Health Organization. WHO-UMC system for standardized case causality assessment. Available from: http://www.who-umc.org/ graphics/4409.pdf. [Last accessed on 2009 Dec 12].

10. Naranjo CA, Busto U, Sellers EM, Sandor P, Ruiz I, Roberts EA, et al. A method for estimating the probability of adverse drug reactions. Clin Pharmacol Ther 1981;30:239-45.

11. Hartwig S, Siegel J, Schneider PJ. Preventability and severity assessment in reporting adverse drug reactions. Am J Hosp Pharm 1992;49:2229-32.

12. Lau PM, Stewart K, Dooley MJ. Comment: Hospital admissions resulting from preventable adverse drug reactions. Ann Pharmacother 2003;37:303-5.

13. Sanchez LA. Pharmacoeconomics. In: Dipiro JT, Talbent RL, editors. Pharmacotherapy. A Pathophysiological Approach. $6^{\text {th }}$ ed. New Delhi: The McGraw-Hill; 2002. p. 1-16.

14. Santos DB, Clavenna A, Bonati M, Coelho HL. Off label and unlicensed drug utilization in Fortaleza, Brazil. Eur J Clin Pharmacol 2008;64:1111-8.

15. Shankar PR, Upadhyay DK, Subish P, Dubey AK, Mishra P. Prescribing patterns among pediatric inpatients in a teaching hospital in western Nepal. Singapore Med J 2006;47:261.

16. Palikhe N. Prescribing pattern of antibiotics in pediatric hospital of Kathmandu valley. J Nepal Health Res Counc 2004;2:31-6.

17. Sawalha A, Al-Bishtawi G, Al-Khaayyat L, Sweileh W, Al-Ramahi R, Jaradat N. Pattern of parenteral antimicrobial prescription among pediatric patients in Al-Watani Government hospital in Palestine. An-Najah Univ J Res (N.Sc.) 2006;20:191-206.

18. Vashishtha VM. Growing antibiotic resistance and need for new antibiotics. Indian Pediatr 2010;47:505-6.

19. Spurling GK, Doust J, Del Mar CB, Eriksson L. Antibiotics for bronchiolitis in children. Cochrane Database Syst Rev 2011;CD005189.

20. Gadomski AM, Brower M. Bronchodilators for bronchiolitis (Review). Cochrane Database Syst Rev 2010;CD001266.

21. Smith SM, Schroeder K, Fahey T. Over-the-counter medications for acute cough in children and adults in ambulatory settings. Cochrane Database Syst Rev 2008;CD001831.

22. Food and Drug Administration. FDA releases recommendations regarding use of over-the-counter cough and cold products. Available from: http://www. fda.gov/NewsEvents/Newsroom/PressAnnouncements/2008/ucm116839. html. [Last updated on 2009 Jun 18; Last accessed on 2009 Aug 16].

23. Salluh J, Povoa P, Soares M, Castro-Faria-Neto HC, Bozza FA, Bozza PT. The role of corticosteroids in severe community-acquired pneumonia: A systematic review. Crit Care 2008;12:R76.

24. Martinez-Mir I, Garcia-Lopez M, Palop V, Ferrer JM, Rubio E, Morales-Olivas FJ. A prospective study of adverse drug reactions in hospitalized patients. Br J Clin Pharmacol 1999;47:681-8.

25. Dos Santos DB, Coelho HL. Adverse drug reactions in hospitalized children in Fortaleza, Brazil. Pharmacoepidemiol Drug Saf 2006;15:635-40.

26. Clavenna A, Bonati M. Adverse drug reactions in childhood: A prospective studies and review of safety alerts. Arch Dis Child 2009;94:724-8.

27. Ehlken B, Ihorst G, Lippert B, Rohwedder A, Peterson G, Schumacher M, et al. Economic impact of community-acquired and nosocomial lower respiratory tract infections in young children in Germany. Eur J Pediatr 2005;164:607-15.

28. Hussain $H$, Waters $H$, Khan AJ, Omer SB, Halsey NA. Economic analysis of childhood pneumonia in northern Pakistan. Health Policy Plan 2008;23:438-42.

29. World Health Organization (WHO). Introduction to drug utilisation research/ WHO International Working Group for Drug Statistics Methodology, WHO Collaborating centre for Drug statistics Methodology, WHO collaborating centre for Drug Utilisation Research and Clinical Pharmacological services. Geneva: WHO press, World Health Organization; 2003.

How to cite this article: Iyer GS, Patel PP, Panchal JR, Dikshit RK. An analysis of the pharmacological management of respiratory tract infections in pediatric in-patients at a tertiary care teaching hospital. Int J Med Public Health 2013;3:140-5.

Source of Support: Nil, Conflict of Interest: None declared. 